\title{
Visibility Graphs of Point Sets in the Plane
}

\author{
Florian Pfender
}

Received: 21 October 2005 / Revised: 23 January 2006

(C) Springer Science+Business Media, LLC 2008

\begin{abstract}
The visibility graph $\mathcal{V}(X)$ of a discrete point set $X \subset \mathbb{R}^{2}$ has vertex set $X$ and an edge $x y$ for every two points $x, y \in X$ whenever there is no other point in $X$ on the line segment between $x$ and $y$. We show that for every graph $G$, there is a point set $X \in \mathbb{R}^{2}$, such that the subgraph of $\mathcal{V}\left(X \cup \mathbb{Z}^{2}\right)$ induced by $X$ is isomorphic to $G$. As a consequence, we show that there are visibility graphs of arbitrary high chromatic number with clique number 6 settling a question by Kára, Pór and Wood.
\end{abstract}

\section{Introduction}

The concept of a visibility graph is widely studied in discrete geometry. You start with a set of objects in some metric space, and the visibility graph of this configuration contains the objects as vertices, and two vertices are connected by an edge if the corresponding objects can "see" each other, i.e., there is a straight line not intersecting any other part of the configuration from one object to the other. Often, there are extra restrictions on the objects and on the direction of the lines of visibility.

Specific classes of visibility graphs which are well studied include bar visibility graphs (see [3]), rectangle visibility graphs (see [6]) and visibility graphs of polygons (see [1]). In this paper we consider visibility graphs of point sets.

Let $X \subset \mathbb{R}^{2}$ be a discrete point set in the plane. The visibility graph of $X$ is the graph $\mathcal{V}(X)$ with vertex set $X$ and edges $x y$ for every two points $x, y \in X$ whenever there is no other point in $X$ on the line segment between $x$ and $y$, i.e., when the point $x$ is visible from the point $y$ and vice versa.

Supported by the DFG Research Center MATHEON (FZT86).

F. Pfender $(\bowtie)$

MA 6-2, TU Berlin, 10623 Berlin, Germany

e-mail: fpfender@math.tu-berlin.de 
Kára et al. discuss these graphs [4], and make some observations regarding the chromatic number $\chi(\mathcal{V}(X))$ and the clique number $\omega(\mathcal{V}(X))$, the order of the largest clique. In particular, they characterize all visibility graphs with $\chi(\mathcal{V}(X))=2$ and $\chi(\mathcal{V}(X))=3$, and in both cases, $\omega(\mathcal{V}(X))=\chi(\mathcal{V}(X))$. Similarly, they show the following proposition.

Proposition 1 Let $\mathbb{Z}^{2}$ be the integer lattice in the plane, then $\omega\left(\mathcal{V}\left(\mathbb{Z}^{2}\right)\right)=$ $\chi\left(\mathcal{V}\left(\mathbb{Z}^{2}\right)\right)=4$.

Note that $\mathcal{V}\left(\mathbb{Z}^{2}\right)$ is not perfect as it contains induced 5-cycles. Further, it is not true in general that $\omega(\mathcal{V}(X))=\chi(\mathcal{V}(X))$-there are point sets with as few as nine points with $\omega(\mathcal{V}(X))=4$ and $\chi(\mathcal{V}(X))=5$.

For general graphs, there are examples with $\chi(G)=k$ and $\omega(G)=2$ for any $k$, one famous example is the sequence of graphs $M_{k-2}$ by Mycielski [5]. No similar construction is known for visibility graphs with a bounded clique number. As their main result, Kára et al. construct a family of point sets with $\chi(\mathcal{V}(X)) \geq$ $\left(c_{1} \log \omega\left(\mathcal{V}\left(X_{i}\right)\right)\right)^{c_{2} \log \omega\left(\mathcal{V}\left(X_{i}\right)\right)}$ for some constants $c_{1}$ and $c_{2}$ and with $\omega\left(\mathcal{V}\left(X_{i}\right)\right)$ getting arbitrarily large. Our main result is the following theorem.

Theorem 2 For every graph $G$, there is a set of points $X \subset \mathbb{R}^{2}$ such that the subgraph of $\mathcal{V}\left(X \cup \mathbb{Z}^{2}\right)$ induced by $X$ is isomorphic to $G$.

Let $G_{k}$ be a graph with $\chi\left(G_{k}\right)=k$ and $\omega\left(G_{k}\right)=2$, and let $X_{k}$ be the corresponding set given by Theorem 2 . Let $Y_{k} \subset X_{k} \cup \mathbb{Z}^{2}$ be the subset of points contained in the convex hull of $X_{k}$. Then $\chi\left(\mathcal{V}\left(Y_{k}\right)\right) \geq \chi\left(G_{k}\right)=k$ and $\omega\left(\mathcal{V}\left(Y_{k}\right)\right) \leq$ $\omega\left(G_{k}\right)+\omega\left(\mathcal{V}\left(\mathbb{Z}^{2}\right)\right)=6$, so we get the following corollary settling the question from above raised by Kára et al.

Corollary 3 For every $k$, there is a finite point set $Y \subset \mathbb{R}^{2}$, such that $\chi(\mathcal{V}(Y)) \geq k$ and $\omega(\mathcal{V}(Y))=6$.

\section{Proof of Theorem 2}

Let $G$ be a graph with vertex set $V(G)=\{1,2, \ldots, n\}$ and edge set $E(G)$. We prove the following lemma in Sect. 3.

Lemma 4 For $M$ large enough, there is a set of prime numbers $\left\{p_{i j}: 1 \leq i<j \leq n\right\}$ with the following properties:

1. $2^{M}<p_{i j}<2^{M+1}$.

2. For $1 \leq k \leq n$, let $P_{k}=2^{n_{k}} \prod_{i=1}^{k-1} p_{i k} \prod_{j=k+1}^{n} p_{k j}$, and choose $n_{k} \in \mathbb{Z}$ such that $\left\lfloor\log _{2} P_{k}\right\rfloor=n M+2 k$. Then $p_{k \ell}$ is the only number in $\left\{p_{i j}: 1 \leq i<j \leq n\right\}$ which divides $P_{\ell}-P_{k}$ for $1 \leq k<\ell \leq n$. 
Note that $\prod_{i=1}^{k-1} p_{i k} \prod_{j=k+1}^{n} p_{k j}<2^{(n-1)(M+1)}<2^{n M}$, and thus $n_{k}>0$ and $P_{k} \in \mathbb{Z}$ for all $k$. From this, we can construct the set of points $X$ in Theorem 2:

$$
X=\left\{x_{i}: 1 \leq i \leq n\right\} \subset \mathbb{R}^{2}, \quad \text { with } x_{i}=\left(2^{-n M} P_{i}, i \frac{\prod_{k<j}\left(P_{j}-P_{k}\right)}{\prod_{k j \in E(G)} p_{k j}}\right)
$$

Before we prove the lemma, we show that this point set has the properties stated in the theorem. For $1 \leq i<\ell \leq n$, let $m_{i \ell}$ be the slope of the line through $x_{i}$ and $x_{\ell}$. Then

$$
m_{i \ell}=\frac{\ell-i}{P_{\ell}-P_{i}} \cdot \frac{2^{n M} \prod_{k<j}\left(P_{j}-P_{k}\right)}{\prod_{k j \in E(G)} p_{k j}} .
$$

There are no three collinear points in $X$, as

$$
2^{n M+2 i+1} \leq P_{i+1}-P_{i}<2^{n M+2 i+3},
$$

thus $m_{i(i+1)}>m_{(i+1)(i+2)}$, and therefore $m_{i \ell}>m_{i k}$ for $i<\ell<k$. Thus, $\mathcal{V}(X)$ is complete, and it remains to show that there is an integer point on the line segment between $x_{i}$ and $x_{\ell}$ if and only if $i \ell \notin E(G)$. To establish this goal, we look at the intersections of the line segment from $x_{i}$ to $x_{\ell}(i<\ell)$ with the integer gridlines parallel to the $y$-axis.

Let $s \in \mathbb{Z}$, with $2^{-n M} P_{i}<s<2^{-n M} P_{\ell}<2^{2 n+1}$. As $2^{2 j} \leq 2^{-n M} P_{j}<2^{2 j+1}$ for every $j$, such an $s$ exists. Let $z_{i \ell}^{s}=\left(s, y_{i \ell}^{s}\right)$ be a point on the line segment from $x_{i}$ to $x_{\ell}$. Then

$$
\begin{aligned}
y_{i \ell}^{s}= & i \frac{\prod_{k<j}\left(P_{j}-P_{k}\right)}{\prod_{k j \in E(G)} p_{k j}}+\left(s-2^{-n M} P_{i}\right) m_{i \ell} \\
= & \underbrace{i \frac{\prod_{k<j}\left(P_{j}-P_{k}\right)}{\prod_{k j \in E(G)} p_{k j}}+s \underbrace{\frac{\ell-i}{P_{\ell}-P_{i}} \cdot \frac{2^{n M} \prod_{k<j}\left(P_{j}-P_{k}\right)}{\prod_{k j \in E(G)} p_{k j}}}_{(2)}}_{(1)} \\
& +\underbrace{P_{i} \frac{\ell-i}{P_{\ell}-P_{i}} \cdot \frac{\prod_{k<j}\left(P_{j}-P_{k}\right)}{\prod_{k j \in E(G)} p_{k j}}} .
\end{aligned}
$$

(3)

Expression (1) is an integer since $p_{k j}$ divides $P_{j}-P_{k}$. By the same argument, (3) is an integer-just note further that $p_{i \ell}$ divides $P_{i}$. It remains the analysis of (2).

If $i \ell \notin E(G)$, then (2) is an integer. Therefore, $z_{i \ell}^{s} \in \mathbb{Z}^{2}$, and $x_{i} x_{\ell} \notin E\left(\mathcal{V}\left(X \cup \mathbb{Z}^{2}\right)\right)$. If $i \ell \in E(G)$, observe that $p_{i \ell}>2^{M}>\max \{\ell-i, s\}$, so $p_{i \ell}$ does not divide $s$ or $\ell-i$. Clearly, $p_{i \ell}$ does not divide $2^{n M}$, and, by Lemma 4 , it does not divide any of the $P_{j}-P_{k}$ other than $P_{\ell}-P_{i}$. Thus, (2) is not an integer, $z_{i \ell}^{s} \notin \mathbb{Z}^{2}$ for all $s$ considered, and $x_{i} x_{\ell} \in E\left(\mathcal{V}\left(X \cup \mathbb{Z}^{2}\right)\right)$, proving Theorem 2 . 


\section{Proof of Lemma 4}

By an inequality of Finsler [2], there are more than $2^{M} /(3(M+1) \ln 2)>2 n^{3}$ prime numbers in the interval from $2^{M}$ to $2^{M+1}$.

We pick the $p_{i j}$ sequentially in the order $p_{12}, p_{13}, \ldots, p_{1 n}, p_{23}, \ldots, p_{(n-1) n}$, with the following conditions given by the lemma:

(a) $p_{i j}$ is a prime number with $2^{M}<p_{i j}<2^{M+1}$.

(b) $p_{i j}$ is different from all the primes picked before.

(c) $p_{i j}$ does not divide $P_{k}-P_{\ell}$ for all $1 \leq \ell<k<i$.

(d) If $j=n$, no $p_{k \ell}$ divides $P_{i}-P_{r}$ for $\{k, \ell\} \neq\{i, r\}$.

Assume that we have picked numbers up to but not including $p_{i j}$ according to (a)-(d), and we want to pick $p_{i j}$. Consider first the case that $j<n$. There were less than $\left(\begin{array}{l}n \\ 2\end{array}\right)$ primes selected before, and each $P_{k}-P_{\ell}$ has at most $n$ prime divisors greater than $2^{M}$, thus at most

$$
\left(\begin{array}{l}
n \\
2
\end{array}\right)+n\left(\begin{array}{l}
n \\
2
\end{array}\right)<n^{3}
$$

of the choices are blocked, and we can find $p_{i j}$ according to (a)-(c).

If $j=n$, pick $p_{i j}$ according to (a)-(c), and assume that $p_{k \ell}$ divides $P_{i}-P_{r}$ for some $\{k, \ell\} \neq\{i, r\}$ (i.e., condition (d) is violated). We have $k \neq i$ as all $p_{i \ell}$ divide $P_{i}$, otherwise $p_{i \ell}$ also divides $P_{r}$ and thus $r=\ell$, a contradiction. Similarly, $\ell \neq i$.

Pick another number $p_{i j}^{\prime}$ according to (a)-(c). If $p_{k \ell}$ divides $P_{i}^{\prime}-P_{r}$, then $p_{k \ell}$ divides $P_{i}^{\prime}-P_{i}=\left(p_{i j}^{\prime}-p_{i j}\right) P_{i} / p_{i j}$, and thus $p_{k \ell}$ divides $p_{i j}^{\prime}-p_{i j}$. However, this is impossible since $\left|p_{i j}^{\prime}-p_{i j}\right|<2^{M}<p_{k \ell}$. Therefore, each $p_{k \ell}$ can block at most one choice for $p_{i j}$ this way, so in total at most $\left(\begin{array}{l}n \\ 2\end{array}\right)$ further choices are blocked by condition (d), and we can always find a number $p_{i j}$ with (a)-(d). This concludes the proof of the lemma.

\section{Further Questions}

We have shown that there are visibility graphs with $\chi(\mathcal{V}(X)) \geq k$ and $\omega(\mathcal{V}(X))=6$ for every $k$. For all visibility graphs with $\omega(\mathcal{V}(X)) \leq 3$, we know that $\chi(\mathcal{V}(X))=$ $\omega(\mathcal{V}(X))$. The only cases left to consider are $\omega(\mathcal{V}(X))=4$ and $\omega(\mathcal{V}(X))=5$. A similar technique of combining a visibility graph with $\omega(\mathcal{V}(X))=3$ with a graph $G$ with $\omega(G)=2$ and a large chromatic number will not work, since the visibility graphs with $\omega(\mathcal{V}(X))=3$ are too simple (all but at most two of their vertices are collinear unless $\mathcal{V}(X)$ is a special graph on six vertices). It would be no surprise to us if the chromatic number of visibility graphs with $\omega(\mathcal{V}(X))=5$ is bounded.

Finally, one could look for smaller point sets with $\chi(\mathcal{V}(X)) \geq k$ and $\omega(\mathcal{V}(X))=6$, as our sets tend to be very large.

\section{References}

1. Abello, J., Kumar, K.: Visibility graphs and oriented matroids. Discrete Comput. Geom. 28, 449-465 (2002) 
2. Finsler, P.: Über die Primzahlen zwischen $n$ und $2 n$. In: Festschrift zum 60. Geburtstag von Prof. Dr. Andreas Speiser, pp. 118-122. Füssli, Zürich (1945)

3. Hutchinson, J.P.: A note on rectilinear and polar visibility graphs. Discrete Appl. Math. 148, 263-272 (2005)

4. Kára, J., Pór, A., Wood, D.R.: On the chromatic number of the visibility graph of a set of points in the plane. Discrete Comput. Geom. 34, 497-506 (2005)

5. Mycielski, J.: Sur le coloriage des graphs. Colloquium Math. 3, 161-162 (1955)

6. Streinu, I., Whitesides, S.: Rectangle visibility graphs: characterization, construction, and compaction. In: Proc. of the STACS 2003. Lecture Notes in Computer Science, vol. 2607, pp. 26-37. Springer, Berlin (2003) 